\title{
Laterality and modality-specific effects of chronic pain
}

\author{
SUZANNE F. SELTZER and MATTHEW YARCZOWER \\ Bryn Mawr College, Bryn Mawr, Pennsylvania \\ and \\ ROBERT WOO and JOSEPH L. SELTZER \\ Jefferson Medical College, Philadelphia, Pennsylvania
}

\begin{abstract}
Previous investigations indicated that thresholds to nonpainful tactile stimuli were elevated in chronic-pain patients when compared with pain-free individuals (Seltzer \& Seltzer, 1986; Seltzer et al., 1988). The present study attempted to determine whether thresholds to tactual and visual stimuli also were elevated by chronic pain. Furthermore, lateralization of the pain effect on tactile thresholds was assessed by obtaining thresholds from both left and right arms. A decrease in tactile sensitivity to nonpainful stimuli in chronic-pain patients was confirmed, but laterality of the effect was not demonstrated. Visual thresholds were not significantly affected by chronic pain. The data in the present study, taken together with other data, support the proposition that pain does not affect right hemispheric processes more than left hemispheric processes.
\end{abstract}

Much of the research on chronic pain in humans has focused on responses to painful stimuli (Chapman et al., 1985). Several investigators have found that pain thresholds in chronic-pain patients are increased, indicating decreased sensitivity to painful stimuli (Callaghan, Sternbach, Nyquist, \& Timmermans, 1978; Merskey \& Evans, 1975). Much less is known about the responses of chronic-pain patients to nonpainful, noninvasive stimuli. One study (Seltzer \& Seltzer, 1986) reported that chronic pain leads to decreased sensitivity to nonpainful tactile stimulation. In that investigation, differences in two-point thresholds were found between chronic-pain patients and pain-free individuals. It also has been demonstrated that relief of pain in the extremities of reflex sympathetic dystrophy (RSD) patients does not decrease the elevated tactual thresholds (Seltzer, Seltzer, Lessin, \& Woo, 1988). This study indicated that the elevated tactual threshold is not an effect that is immediately reversed when pain relief is obtained. The present study attempted to determine whether the decreased sensitivity brought about by pain also extended to a tactual and a visual task, specifically depth perception.

Several factors entered into the decision to use a depthperception task. First, it had been shown, using a depthperception task, that there were more correct detections of depth in the left visual field than in the right visual field (Grabowska, 1983). Furthermore, Carmon and Bechtoldt (1969) found that depth perception was worse in patients with lesions in the right hemisphere than in patients with

Appreciation is expressed to Alexander T. Marr, CRNA, for his assistance in the data collection. Correspondence should be addressed to S. F. Seltzer, Graduate School of Arts and Sciences, Department of Psychology, Bryn Mawr College, Bryn Mawr, PA 19010. lesions in the left hemisphere. These results suggested greater involvement of the right hemisphere than the left hemisphere in depth perception.

Second, some investigations have reported that pain thresholds were lower for the left hand than for the right hand in individuals who were initially free of pain (Haslam, 1970; Murray \& Safferstone, 1970). The latter investigation indicated that the left hand was more sensitive than was the right hand for both threshold and tolerance measures using a cold-pressor method of experimental-pain induction. Weinstein and Sersen (1961) tested pain-free individuals and demonstrated that for right-handed individuals, pressure thresholds were lower on the left side than on the right side of the body. Wolff and Jarvik (1964) studied chronic arthritic patients and found consistent differences in thresholds between left and right hands using cold-pressor, radiant-heat, and chemical methods for induction of the experimental pain. Their results indicated that the left hand was more sensitive to pain. These authors speculated that the dominant hemisphere may be more sensitive to pain or more closely integrated with an individual's emotional behavior than is the nondominant hemisphere.

Additionally, Merskey and Watson (1979) suggested that there is greater sensitivity to pain on the left side than on the right side of the body. Furthermore, when pain was lateralized, they found that individuals reported that it occurred more frequently on the left side of the body. They suggested that this may be due to qualitative differences between left and right hemispheres in their capacity to integrate and discriminate sensory input. Supporting this proposition was a recent investigation that administered an EEG power-spectrum analysis to chronic-pain patients (DeBenedittis \& DeGonda, 1985). The results of this in- 
vestigation revealed that there was a tendency toward less left hemispheric dominance during mathematical and visuospatial tasks in psychogenic-pain patients than in pain-free individuals. Chronic pain, particularly psychogenic pain, has been associated with emotional disturbances such as depression, and pain is the most common somatic complaint among psychiatric patients (Merskey \& Watson, 1979). It is known that emotional processes affect the perception of pain (Merskey \& Evans, 1975) and that the right hemisphere may be dominant for the negative emotions so often associated with pain (Ahern \& Schwartz, 1985; Tucker, 1981).

There is, however, conflicting evidence concerning lateralization of symptoms in chronic pain (Campbell, Lahverta, \& Bowsher, 1985; Hall, Hayward, \& Chapman, 1981; Lam \& Remick, 1988; Margolis, Krause, \& Tait, 1985). In contrast to the studies cited above, these studies, based upon self-reports of patients, found no evidence that pain occurred more frequently on one side than on the other side of the body.

Thus, there is some support, albeit somewhat inconsistent, for the suggestion that there is greater involvement of the right hemisphere in pain as well as in depth perception. If chronic pain is more likely to affect activities lateralized in the right hemisphere, and if the right hemisphere is importantly involved in depth perception, then perhaps chronic pain would affect depth perception.

The present study used a depth-perception task to assess the effects of chronic pain. Furthermore, although a previous study (Seltzer \& Seltzer, 1986) found decreased sensitivity in chronic-pain patients to nonpainful tactual stimuli, their results were obtained using only the right arm. The present study attempted to replicate the previous finding of decreased sensitivity in chronic-pain patients and at the same time to assess whether the threshold difference between chronic-pain patients and pain-free control individuals is different for the left and right arms.

\section{METHOD}

\section{Subjects}

There were 20 right-handed (by self-report) chronic-pain patients and 20 right-handed (by self-report) individuals in a pain-free control group, for a total of $\mathbf{4 0}$ subjects, who were matched by age and sex. The criterion for inclusion in the control group was a lack of a report of any type of pain. The sample of patients tested were those who were referred to the Thomas Jefferson University Hospital Pain Clinic by their personal physicians for treatment or diagnosis related to chronic pain.

Six patients were using prescription drugs at the time of testing The data for these 6 patients and their matched controls were not included in the analyses of the data.

\section{Apparatus}

The depth-perception task was performed using a Layfayette Instruments modified Howard-Dolman apparatus (Model 14012). The unit is constructed so that there are no external depth cues; depth discrimination is based solely on binocular cues. A Layfayette Instruments three-point aesthesiometer (Model 1707744) was used for the two-point threshold testing. Black goggles were worn by the subjects during the two-point threshold testing to prevent visual detection of aesthesiometer distance.

\section{Procedure}

This study was approved by the Jefferson Medical College Institutional Review Board. A nurse anesthetist performed the testing of all the subjects and was not aware of the expectations of the outcome of the study. Each subject was seen individually by the experimenter, and a questionnaire about age, duration of pain, medications, and medical diagnoses was completed prior to the testing. The procedure was explained to the subjects in both groups prior to the request for their consent, and at the same time they were told that the testing procedures were not painful. The depthperception testing took place first. If corrective lenses were worn, then the subjects were asked to wear them during the depthperception testing. Each subject was seated at a distance of $2.4 \mathrm{~m}$ from the Howard-Dolman apparatus. Measurements were taken to determine eye-level distance from the floor so that the apparatus was positioned at eye level for all subjects. The rods in the apparatus were moved to their most extreme forward position and to their most extreme distant position during the familiarization period, which also served as the partial run (Bradley, 1977). The adjustments were made by maneuvering a length of string attached to the rods inside the apparatus. The subject was asked to lay the string down between each rod adjustment and to close his/her eyes while the adjustment was being made. The rods were set 20 times at randomly selected positions between +6 and $-6 \mathrm{~cm}, 0$ being the point defining perfect adjustment. The subject was asked to adjust them until they appeared to be aligned. Absolute differences in distance between the 2 rods were recorded, and the mean absolute error for each subject was calculated.

The two-point threshold testing was performed next. The subject remained seated in the same chair and then was blindfolded. The hand to be tested was laid flat on the table with the palm up. The skin area used for testing was the lateral side of the dorsal forearm. Which arm was used was alternated with each subject. For example, the first subject presented his/her right arm first, the second subject, his/her left arm first, and so forth. The subject was told: "You will feel one or two points of stimulation on your forearm. Reply by saying 'one,' or 'two,' as you feel it."

There were four sequences with a series of two alternating ascending and descending numbers presented in increments of $2 \mathrm{~mm}$, performed on both the right and left forearms. The initial distance for both the ascending and descending sequences was arbitrarily selected. The ascending stimulus began well below the threshold level, and the descending began well above the threshold level. On the ascending sequences, the stimulus presented was a slight pressure on the forearm with the two points of the aesthesiometer. The interstimulus interval was approximately $10 \mathrm{sec}$. As the distance between the two points of stimulation was increased, the subject sometimes reported uncertainty but was asked to make a decision between one and two points. The investigator stopped the sequence when a distance was reached where the subject reported two distinct points of stimulation. The same procedure was used for the descending sequences, but the sequence was stopped when the subject reported a sensation of one point. The ascending series began with $8 \mathrm{~mm}$ and $12 \mathrm{~mm}$. The descending series began with $44 \mathrm{~mm}$ and $46 \mathrm{~mm}$. The length of the testing session varied with the sensitivity of the subject but was approximately $20 \mathrm{~min}$ in length. The threshold was defined as the midpoint, in millimeters, of the points where a transition occurred from one response to another. A "catch trial" "occasionally was introduced in which the subject's forearm was deliberately touched by one point of the aesthesiometer. This was used to determine if there was any response bias.

\section{RESULTS}

One patient responded incorrectly on $75 \%$ of the catch trials. That patient's data and the data for the matched control subject were eliminated from further considera- 
tion. The mean percent correct responses on the catch trials for the patient and control groups were $96.9 \%$ and $93.1 \%$, respectively. There was no significant difference between the two means $(p>.05)$.

A $t$ test for related measures, based on the 13 matched pairs of patients and controls, was conducted on the data for two-point thresholds. There was a significant difference in the two-point thresholds between the patient and control groups $[t(12)=2.78, p<.02]$. The mean threshold for the patient group was $38.94 \mathrm{~mm}$ and for the control group was $30.85 \mathrm{~mm}$.

The difference between thresholds obtained from the left and right arms was not statistically significant for the patient group $[t(12)=1.98, p>.05]$ or for the control group $[t(12)=0.06, p>.05]$. The mean thresholds for the patient group were $35.32 \mathrm{~mm}$ for the left arm and $42.56 \mathrm{~mm}$ for the right arm. The mean thresholds for the control group were $28.88 \mathrm{~mm}$ for the left arm and $32.82 \mathrm{~mm}$ for the right arm.

A $t$ test for related measures of the depth-perception data revealed nonsignificant differences in the absolute errors between patient and control groups $[t(12)=0.30$, $p>.05]$. The mean errors were $1.75 \mathrm{~cm}$ for the patient group and $1.71 \mathrm{~cm}$ for the control group.

\section{DISCUSSION}

This study confirmed the results of a previous study that found that two-point thresholds were higher for chronicpain patients than for a control sample of pain-free individuals (Seltzer \& Seltzer, 1986). The results of the present study also indicated that the effects of chronic pain do not extend to all sensory modalities; visual depth perception was not affected by chronic pain.

Chronic pain decreases sensitivity to tactile stimuli but not to visual stimuli. This differential effect on tactual and visual modalities may be due to the fact that activation of different receptors are involved in the processing of these two sensory modalities. Pain is mediated by nociceptors, and nonpainful tactual sensation is mediated by mechanoreceptors (Lindblom, 1965; Mountcastle, La Motte, \& Carli, 1972). Central processing of nociceptive responses may interfere with the processing and transmission of nonpainful tactile stimuli. This may be due in part to simultaneous firing of both the nociceptors involved in the chronic-pain process and the mechanoreceptors involved in the transmission of the nonpainful tactile stimulation.

Furthermore, visual stimuli, which of course do not involve mechanoreceptors, remained unaffected by chronic pain. Neither the depth-perception data nor the two-pointthreshold data support the proposition that chronic pain influences the right hemispheric processes more than the left hemispheric processes. It also would appear that chronic pain leads to specific deficits in particular sensory modalities. In fact, it may be possible that chronic pain leads to more specific deficits even within the tactual modality. Grushka, Sessle, and Howley (1987) reported no significant differences between individuals with burn- ing mouth syndrome (BMS) and matched controls on tactile thresholds, detection of thermal change, intensity ratings of thermal stimuli, and heat-pain thresholds. These measures were obtained from the tongue, palate, lip, and cheek areas.

It may well be that sensory deficits of chronic pain not only are modality specific, but that even within the tactual modality, the deficit depends upon the region of the body. Of course, the findings of Grushka et al. (1987) may apply only to pain arising from BMS. It would be interesting to know whether two-point thresholds obtained from the arm would yield deficits due to BMS pain.

If there is greater involvement by the right hemisphere than by the left hemisphere in tactile sensitivity, then one would have expected lower two-point thresholds for the left arm than for the right arm in pain-free individuals. However, the present results revealed no significant differences in thresholds between the left and right arms in the control sample. The current data also do not support some of the suggestions in the literature that pain effects are lateralized in the right hemisphere. These suggestions would lead to the expectation of higher two-point discriminations on the left side of the body in chronic-pain patients.

When we analyzed the data presented by Grushka et al. (1987, Table 2), we found that the differences in the mean two-point thresholds for the left and right cheeks were nonsignificant for BMS patients $[t(28)=0.40, p>.05]$ as well as for the control sample $[t(34)=0.78, p>.05]$. (There also were nonsignificant differences in the twopoint thresholds for the left and right sides of the tongue, but since there is bilateral innervation of the tongue, one should not expect to find asymmetry.) Thus, these data are consistent with the results of the present study in not revealing any asymmetry in two-point thresholds.

The rationale for expecting right hemispheric involvement during a painful experience was the suggestion that pain contains a strong emotional component and that emotional processes are lateralized and predominantly located in the right hemisphere (Borod \& Caron, 1980; Sacheim \& Gur, 1978; Schwartz, Davidson, \& Maer, 1975). However, the concept that emotions are located predominantly in the right hemisphere has been challenged recently by investigators who have suggested that different types of emotion may involve different cerebral hemispheres (Davidson \& Fox, 1982; Reuter-Lorenz \& Davidson, 1981), that is, positive and negative emotions may be lateralized in different hemispheres. Furthermore, Hager and Ekman (1985) have even questioned whether there is any lateralization of genuine expressions of emotion. They suggested that previous studies, which had concluded that there was laterality of emotion, had not differentiated between emotional and nonemotional processes. In their study, elicited, genuine, and spontaneous emotional facial expressions were shown to be symmetrical. Simulated, or voluntarily regulated, facial expressions were asymmetrical. The study of the laterality of emotional expressions must be accompanied by data that indicate whether genuine or simulated emotions are involved. 
These two different types of emotional expressions differ in the involvement of pyramidal and nonpyramidal pathways and may not involve the cerebral hemispheres in the same way (Hall et al., 1981).

Although pain may engender emotional processes and although emotional processes may be lateralized, the data in the present study do not support the proposition that there is lateralization of sensory effects of pain.

\section{REFERENCES}

Ahern, G. L., \& Schwartz, G. E. (1985). Differential lateralization for positive and negative emotion in the human brain: EEG spectral analysis. Neuropsychologia, 23, 745-755.

Borod, J. C., CARON, H. S. (1980). Facedness and emotion related to lateral dominance, sex and expression type. Neuropsychologia, 18, 237-241.

Bradley, B. C. (1977). Effective measures with a modified HowardDolman apparatus. Perceptual \& Motor Skills, 44, 1335-1341

Callaghan, M., Sternach, R. A., Nyquist, J. K., \& Timmermans, G. (1978). Changes in somatic sensitivity during transcutaneous electrical analgesia. Pain, 5, 115-127.

Campbell, J. A., LAhuerta, J., \& Bowsher, D. (1985). Pain laterality in relation to site of pain and diagnosis. Pain, 23, 61-66.

Carmon, A., \& Bechtoldt, H. P. (1969). Dominance of the right cerebral hemisphere for stereopsis. Neuropsychologia, 7, 29-39.

Chapman, C. R., Casey, K. L., Dubner, R., Foley, K. M., Gracely, A. H., Reading, A. E. (1985). Pain measurement: An overview. Pain, 22, 1-30.

Davidson, R. J., * Fox, N. A. (1982). Asymmetrical brain activity discriminates between positive and negative stimuli in human infants. Science, 218, 1235-1237.

DeBeneditTis, G., \& DeGondA, F. (1985). Hemispheric specialization and the perception of pain: A task related EEG power spectrum analysis in chronic pain patients. Pain, 22, 375-384.

GrABOWSKA, A. (1983). Lateral differences in the detection of stereoscopic depth. Neuropsychologia, 21, 49-57.

Grushka, M., Sessle, B. M., Howley, T. P. (1987). Psychophysical assessment of tactile, pain and thermal sensory functions in burning mouth syndrome. Pain, 28, 169-184.

Hager, J. C., E Ekman, P. (1985). The asymmetry of facial actions is inconsistent with models of hemispheric specialization. Psychophysiology, 22, 307-318.
Hall, W., Hayward, L., Chapman, C. R. (1981). On the lateralization of pain. Pain, 10, 337-351.

HASLAM, D. R. (1970). Lateral dominance in the perception of size and pain. Quarterly Joumal of Experimental Psychology, 22, 503-507.

LAM, R. W., Remick, R. A. (1988). The lateralization of atypical facial pain. Canadian Journal of Psychiarry, 33, 100-102.

LINDBLOM, U. (1965). Properties of touch receptors in distal glabrous skin of the monkey. Jourmal of Neurophysiology, 29, 966-985.

MArgolis, R. B., Krause, S. J., \& TAIT, R. C. (1985). Lateralization of chronic pain. Pain, 23, 289-293.

Merskey, H., Evans, P. R. (1975). Variations in pain complaint threshold in psychiatric and neurologic patients with pain. Pain, 1 , 73-79.

Merskey, H., WAtson, G. D. (1979). The lateralization of pain Pain, 7, 271-280.

Mountcastle, V. B., LAMotte, R. H., CARL, G. (1972). Detection thresholds for stimuli in humans and monkeys: Comparison with threshold events in mechanoreceptive afferent nerve fibers innervating the monkey hand. Joumal of Neurophysiology, 35, 122-136.

Murray, F. S., SAFFerstone, J. F. (1970). Pain threshold and tolerance of right and left hands. Joumal of Comparative \& Physiological Psychology, 71, 83-86.

Reuter-Lorenz, P., Davidson, R. S. (1981). Differential contributions of the two cerebral hemispheres to the perception of happy and sad faces. Neuropsychologia, 19, 609-613.

SaCheim, H. A., \& GUR, R. C. (1978). Lateral asymmetry in intensity of emotional expression. Neuropsychologia, 16, 473-481.

SChwartz, G. E., Davidson, R. J., \& MAER, F. (1975). Right hemisphere lateralization for emotion in the human brain: Interaction of cognition. Science, 190, 286-288.

Seltzer, S. F., Seltzer, J. L. (1986). Tactual sensitivity of chronic pain patients to non-painful stimuli. Pain, 27, 291-295

Seltzer, S. F., Seltzer, J. L., Lessin, J., Woo, R. (1988). Sympathetic block does not improve tactile sensitivity in patients with reflex sympathetic dystrophy. Anesthesiology, 69, A714.

TUCKER, D. M. (1981). Lateral brain function, emotion, and conceptualization. Psychological Bulletin, 89, 19-46.

Weinstein, S., \& Sersen, E. A. (1961). Tactual sensitivity as a function of handedness and laterality. Journal of Comparative \& Physiological Psychology, 54, 665-669.

WolfF, B. B., JARVIK, M. E. (1964). Relationship between superficial and deep somatic thresholds of pain with a note on handedness. American Joumal of Psychology, 77, 589-599.

(Manuscript received July 30, 1990; revision accepted for publication January 2, 1992.) 